\title{
Importance of the Use of the Appropriate Pharmacokinetic Model to Analyze In Vivo Enzyme Constants
}

\author{
Allen J. Sedman ${ }^{1}$ and John G. Wagner ${ }^{1,2}$ \\ Received Sept. 4, 1973-Final Jan. 18, 1974
}

Throughout the literature, enzyme constants have been derived by utilizing in vivo data and indirectly assuming that these data were described by the one-compartment open model. Howeter, many drugs are probably best described by a two-compartment open model with Michaelis-Menten elimination kineties. Simulated data, which obey the two-compartment open model with MichaetisMenten elimination, and which illustrate some of the interesting properties of such models, are presented. Treatment of two-compartment data by one-compartment analysis is shown to result in a serious distortion of enzyme parameters $\left(V_{m}, K_{m}\right)$. For data which obey the two-compartment open model, estimation of the Michaelis-Menten constant $\left(K_{m}\right)$ and the maximum velocity $\left(V_{m}\right)$ by one-compartment analysis cannot be theoretically justified and therefore should be avoided.

KEY WORDS: Michaelis-Menten kinetics; dose-dependent kinetics; one-compartment model; two-compartment model.

\section{INTRODUCTION}

The classical linear pharmacokinetic models have been successfully used to accurately describe rates of absorption, metabolism, and excretion when applied to certain specific drugs and have been very useful for predictive purposes. During the past decade, many reports have provided evidence that linear systems of differential equations do not accurately describe the pharmacokinetic behavior of many compounds. Nonlinear pharmacokinetics to date are based on the Michaelis-Menten and Langmuir

Supported in part by Public Health Service Grant 5-P11-GM15559.

${ }^{1}$ College of Pharmacy and Upjohn Center for Clinical Pharmacology, The University of Michigan, Ann Arbor, Michigan 48104.

${ }^{2}$ Address requests for reprints to Dr. John G. Wagner, Upjohn Center for Clinical Pharmacology, The University of Michigan, Ann Arbor, Michigan 48104. 
tissue-binding equations. Several authors (1-4) have successfully used nonlinear pharmacokinetic models involving Michaelis-Menten equations to describe drug concentration-time data. However, these authors obtained values for the constants, $V_{m}$ and $K_{m}$, either from separate experiments (e.g., from in vitro studies) or by fitting data directly to the one-compartment open model with Michaelis-Menten elimination. Many of the methods used to indirectly evaluate enzyme constants in animals cannot be used in human beings. In addition, other authors (5-6) have shown that in vitro enzyme constants are markedly dependent on experimental conditions. This sensitivity can introduce a large amount of uncertainty into the values of enzyme constants obtained from in vitro studies because in vitro enzyme environment may not accurately simulate conditions existing in vivo.

Direct pharmacokinetic estimation of enzyme constants avoids the problems above, but, as will be demonstrated, correct evaluation of these constants requires the use of an appropriate pharmacokinetic model.

Dedrick and Forrester (7) discussed the errors introduced into the values of $V_{m}$ and $K_{m}$ when data described by a special form of the twocompartment model were analyzed according to the one-compartment open model.

Wagner and Patel (3) used the one-compartment open model to analyze enzyme constants describing ethanol metabolism in man. Their data indicate the presence of a distribution phase, which is particularly evident after the administration of $60 \mathrm{ml}$ of $95 \%$ ethanol. This suggests that a two-compartment model involving Michaelis-Menten elimination may prove to be a more accurate representation of pharmacokinetics of ethanol in man, and the remarks made by Dedrick and Forrester may apply. Therefore, the recognition of data described by multicompartment models and the consequences of analyzing such data by use of the onecompartment model are of considerable interest and importance.

The purposes of this paper are:

1. To discuss some of the properties of the two-compartment open model with Michaelis-Menten elimination.

2. To demonstrate the effects of fitting data which were generated with the equations of a two-compartment model involving Michaelis-Menten elimination kinetics by using the equations of a one-compartment model with Michaelis--Menten elimination kinetics.

\section{THEORY}

A two-compartment open model involving first-order absorption and Michaelis Menten elimination from the central compartment is shown schematically in scheme 1 : 


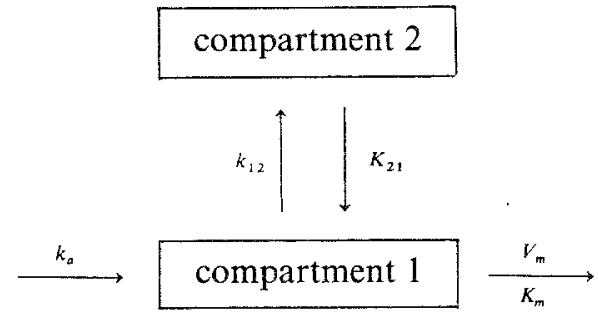

The model can be described mathematically by the following differential equations:

$$
\begin{gathered}
\frac{d C_{1}}{d t}=k_{a} C_{0} e^{-k_{a} t}-\left(k_{12}+\frac{V_{m}}{K_{m}+C_{1}}\right) C_{1}+k_{21} C_{2} \\
\frac{d C_{2}}{d t}=k_{12} C_{1}-k_{21} C_{2}
\end{gathered}
$$

where $C_{0}$ is the dose of drug/volume of compartment $1(\mathrm{mg} / \mathrm{ml}), C_{1}$ is the concentration of drug in compartment $1(\mathrm{mg} / \mathrm{ml}), C_{2}$ is the amount of drug in compartment $2 /$ volume of compartment $1(\mathrm{mg} / \mathrm{ml}), t$ is time (hr), $k_{a}$ is the first-order absorption rate constant $\left(\mathrm{hr}^{-1}\right), k_{12}$ is the first-order distribution rate constant from compartment 1 to compartment $2\left(\mathrm{hr}^{-1}\right), k_{21}$ is the first-order distribution rate constant from compartment 2 to compartment 1 $\left(\mathrm{hr}^{-1}\right), V_{m}$ is the maximum reaction rate $[\mathrm{mg} /(\mathrm{ml})(\mathrm{hr})]$, and $K_{m}$ is the Michaelis constant $(\mathrm{mg} / \mathrm{ml})$.

A one-compartment model with Michaelis-Menten elimination is shown in scheme 2 :

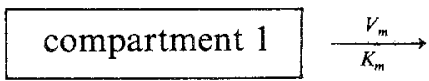

The model is mathematically described by

$$
-\frac{d C_{1}}{d t}=\frac{V_{m} \cdot C_{1}}{K_{m}+C_{1}}
$$

where the symbols are defined as above.

These equations can be used to obtain values of $C_{1}$ and/or $C_{2}$ as a function of time by numerical integration. They also may be used in a similar manner to fit drug concentration-time data by means of a nonlinear least-squares estimation program and a high-speed digital computer.

\section{EXPERIMENTAL}

\section{Generation of Simulated $C, t$ Data}

Simulated $C, t$ data obeying equations 1 and 2 were generated by assigning numerical values to $C_{0}, k_{a}, k_{12}, k_{21}, V_{m}$, and $K_{m}$ using the program NONLIN and an IBM 360/67 computer. Equations 1 and 2 were numerically 
integrated by the computer using the Runge-Kutta method, which is a part of the program NONLIN. The simulations were performed with $V_{m}=0.418 ; K_{m}=0.182 ; k_{12}=3 ; k_{21}=2 ; k_{a}=8,4,2,1$, and $0.5 ; C_{0}=3$, 2 , and 0.5 .

\section{Fitting of Simulated $C, t$ Data}

The terminal portions of all of the sets of simulated data were fitted to the one-component open model with Michaelis-Menten elimination kinetics (scheme 2) by utilizing a linear transformation of equation 3 . This technique is discussed in the Appendix. The constants for one set of data ( $k_{a}=8$ and $C_{0}=2$ ) were also evaluated by two additional means: (a) a Lineweaver-Burk plot (8) of the terminal data and (b) nonlinear leastsquares computer fitting of the terminal data to equation 3 .

\section{RESULTS AND DISCUSSION}

Some of the $C, t$ data generated as described in the Experimental section are plotted on cartesian coordinate graphs in Figs. 1 and 2. These curves are characterized by an early distribution phase (a "nose" evident at the higher doses) followed by a pseudolinear region, then terminating in a curved portion. The presence of the distribution phase, indicated by the steeper slope of the blood concentration curve just past the peak, becomes increasingly apparent as the absorption rate and/or the dose of drug are raised. The slope of the subsequent pseudolinear phase $\left(k_{0}\right)$ is markedly dependent on both the absorption rate and the dose of drug (Fig. 3). The magnitude of $k_{0}$ is only a small percentage of the maximum velocity, $V_{m}$, and therefore does not represent the maximum metabolic rate of the drug. The value of this slope, alone, cannot be used to estimate any of the enzyme parameters. As illustrated by Figs. 4 and 5, the peak drug concentration and the areas under the $C, t$ curves $(0-6 \mathrm{hr})$ are both dependent on the rate of drug absorption and the dose of drug. Although not shown, the relationships of total area $(0-\infty \mathrm{hr})$ under the $C, t$ curves to $C_{0}$ and $k_{a}$ are very similar to those shown in Fig. 5. Slowing the absorption rate of a drug which is fully absorbed not only decreases the peak drug concentration but also decreases the area under the $C, t$ curve. These phenomena would also be predicted by the one-compartment open model with first-order absorption and Michaelis-Menten elimination, but could not be explained by the classical linear one- or two-compartment open models. Either nonlinear model could help explain the effects of food and stomach emptying on drug blood levels and bioavailability. Even for drugs which are fully absorbed, these models would predict decreases in drug blood concentration and 


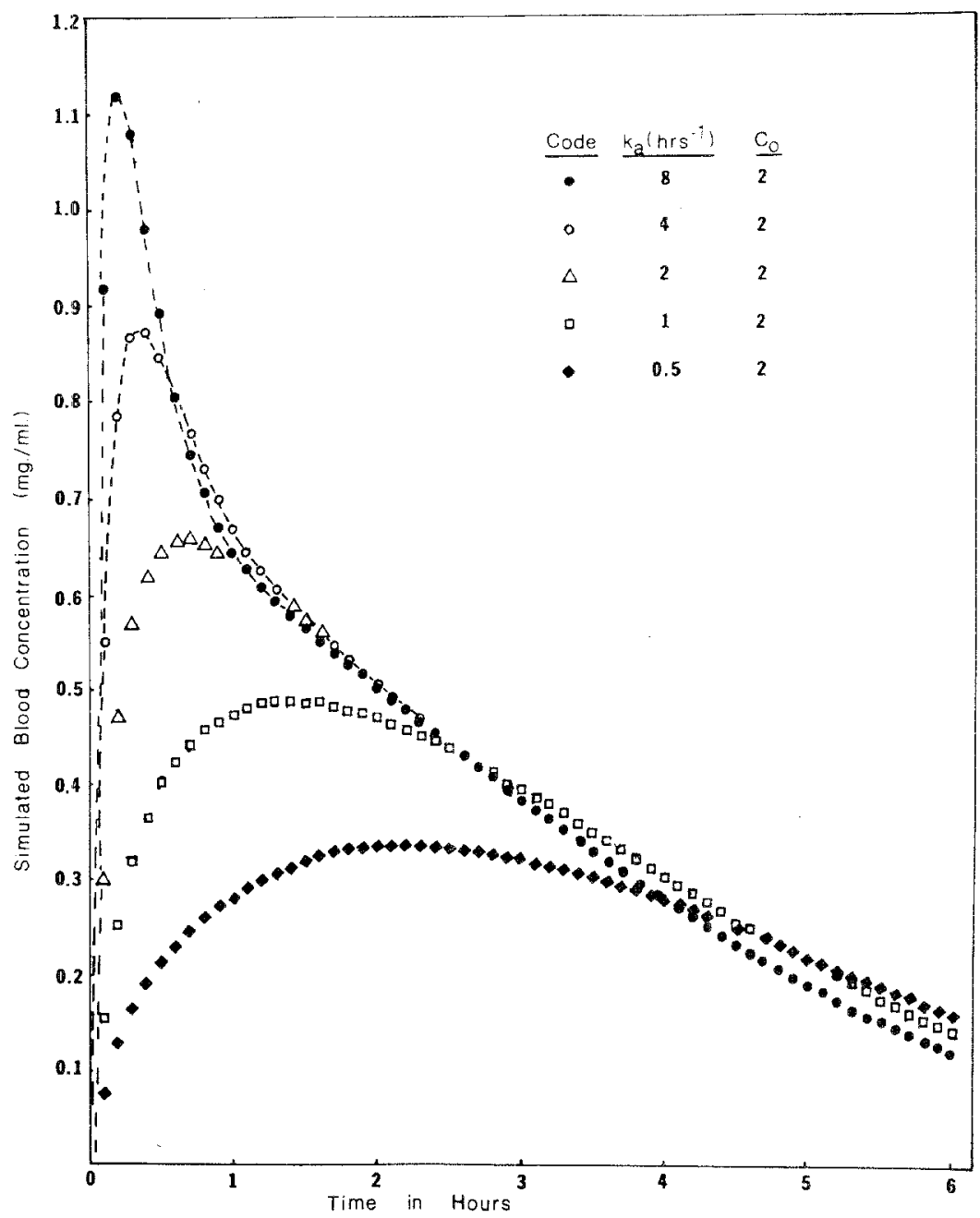

Fig. 1. Effect of drug absorption rate on simulated concentration data when data were generated from the two-compartment open model (scheme 1) with $k_{12}=3, k_{21}=2$, $V_{m}=0.418$, and $K_{m}=0.182$.

bioavailability when food was administered or stomach emptying was slowed because of a decreased rate of drug absorption.

The values listed in Table I demonstrate the effect of using the onecompartment open model (scheme 2) to fit data which were generated using the two-compartment open model (scheme 1). In order to compare the procedure outlined in the Appendix with more commonly used methods, the enzyme constants for one set of data $\left(k_{a}=8, C_{0}=2\right)$ were also analyzed 


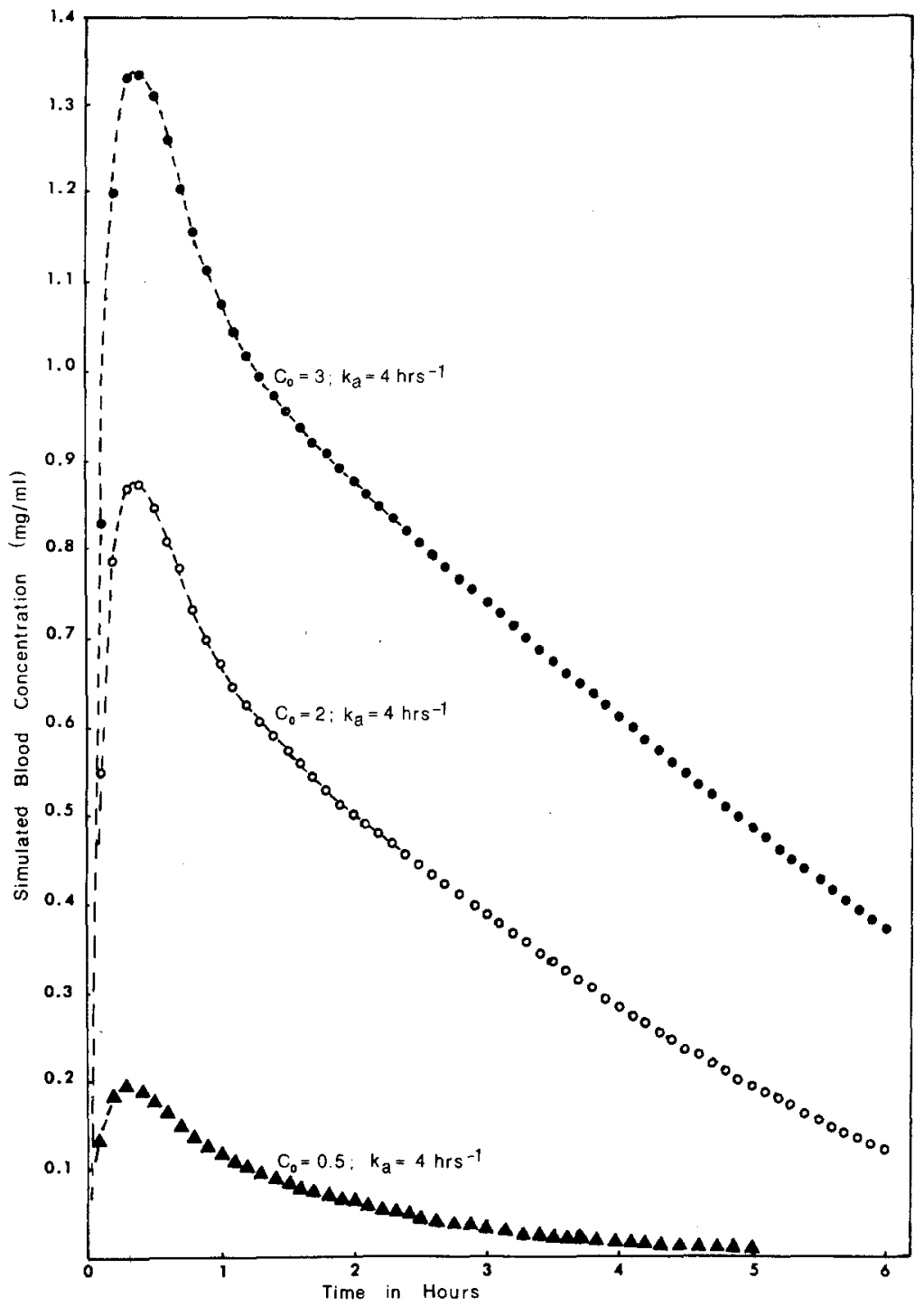

Fig. 2. Effect of change in dose $\left(C_{0}\right.$ value $)$ on simulated concentration data when data were generated from the two-compartment open model (scheme 1) with $k_{12}=3$, $k_{21}=2, V_{m}=0.418, K_{m}=0.182$, and $k_{a}=4$. 


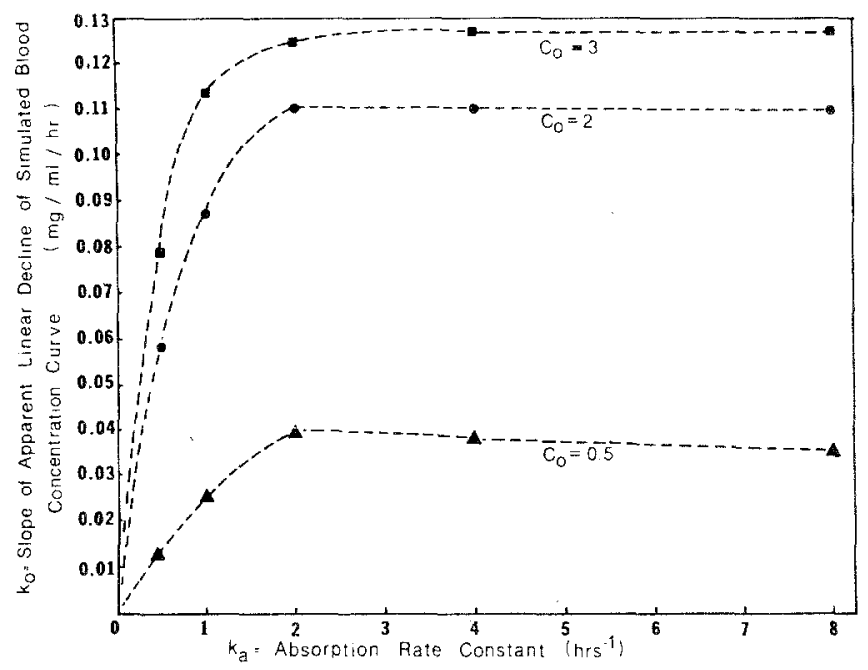

Fig. 3. Effect of dose and drug absorption rate on the slope of the apparently linear decline of the simulated blood concentration curve.

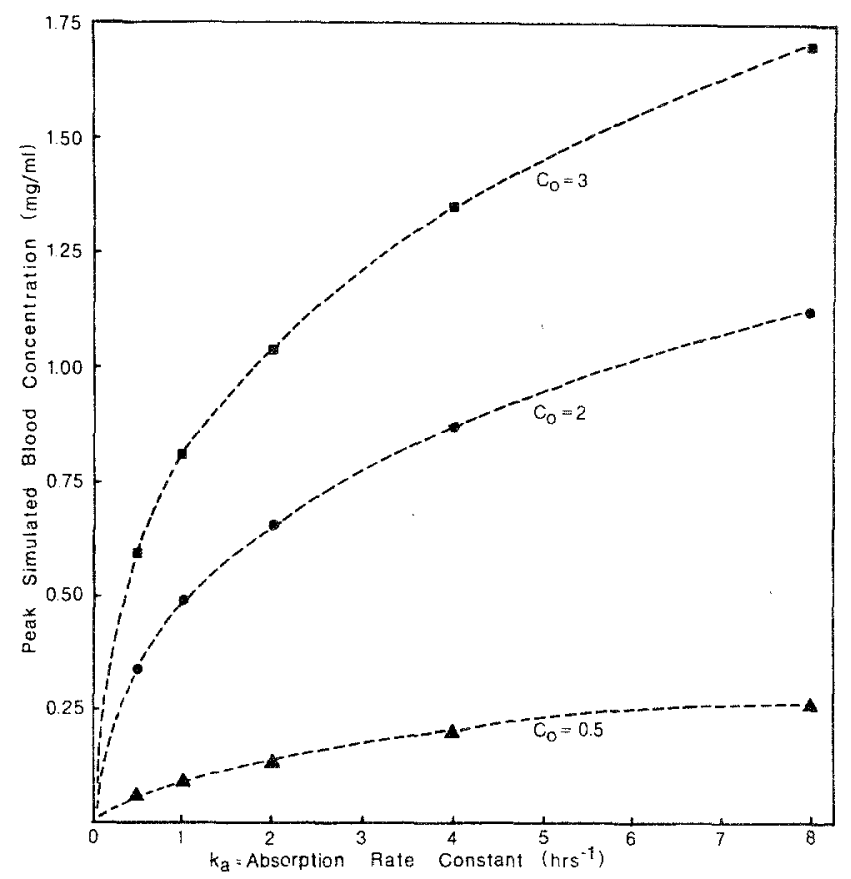

Fig. 4. Effect of drug absorption rate and dose on the peak simulated blood concentration. 


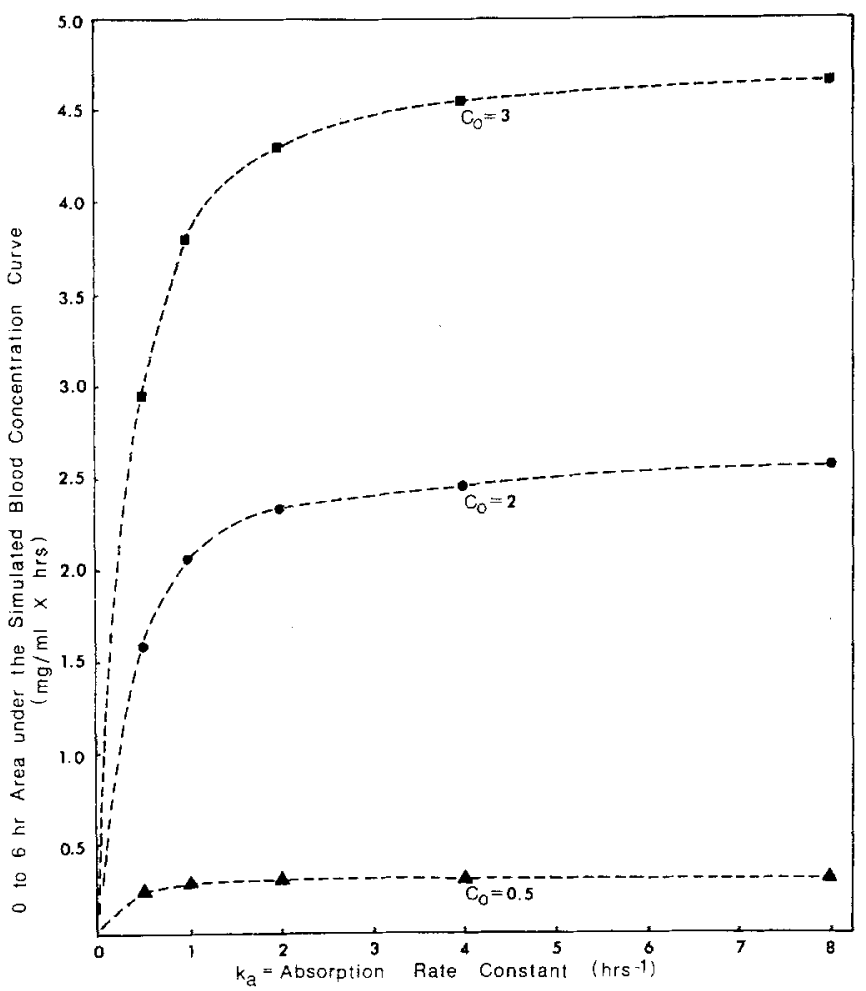

Fig. 5. Effect of drug absorption rate and dose on the $0-6 \mathrm{hr}$ area under the simulated blood concentration curve.

by constructing a Lineweaver-Burk plot (8) from the terminal data (Fig. 6 ) and by nonlinear least-squares computer fitting of the terminal data to the Michaelis-Menten equation (equation 3). The former method yielded values of $V_{m}=0.180$ and $K_{m}=0.238$. Values of $V_{m}=0.176$ and $K_{m}=0.227$ were obtained from the computer fitting. Both sets of estimates agree reasonably well with those listed in Table I. As illustrated in Table I, the following are observed when two-compartment data are fitted to a one-compartment model: (a) Enzyme constants estimated in such a manner bear little relationship to the values of the kinetic constants used in the simulations. Moreover, the estimated values of the constants are dependent on the drug absorption rate $\left(k_{a}\right)$ and the dose of drug. (b) The value of $V_{m} / K_{m}$ estimated by use of the one-compartment open model is always much less than the value used to generate the two-compartment open model data. It is not surprising that the values of $V_{m} / K_{m}$ obtained by the fitting of simulated data to the one- 
Table I. $V_{m}$ and $K_{m}$ Values Estimated from Terminal Simulated Concentration Data According to One-Compartment Open Model (scheme 2) Analysis When Data Were Generated from the Two-Compartment Open Model (scheme 1) with $k_{12}=3, k_{21}=2, V_{m}=0.418$, and $K_{m}=0.182$

\begin{tabular}{|c|c|c|c|c|}
\hline \multirow{2}{*}{$\begin{array}{c}C_{0} \\
(\mathrm{mg} / \mathrm{ml})\end{array}$} & \multirow{2}{*}{$\begin{array}{c}k_{a} \\
\left(h r^{-1}\right)\end{array}$} & \multirow{2}{*}{$\begin{array}{l}\text { Time interval }{ }^{a} \\
(\mathrm{hr})\end{array}$} & \multicolumn{2}{|c|}{ One-compartment model estimates } \\
\hline & & & $V_{m}$ & $K_{m}$ \\
\hline 3 & 8 & $3.6-9.6$ & 0.174 & 0.223 \\
\hline 3 & 4 & $3.6-9.6$ & 0.174 & 0.223 \\
\hline 3 & 2 & $3.6-9.6$ & 0.174 & 0.223 \\
\hline 3 & 1 & $4.5-10.0$ & 0.163 & 0.200 \\
\hline 3 & 0.5 & $6.5-10.5$ & 0.134 & 0.162 \\
\hline 2 & 8 & $1.8-8.3$ & 0.179 & 0.239 \\
\hline 2 & 4 & $1.8-8.3$ & 0.184 & 0.248 \\
\hline 2 & 2 & $1.8-8.3$ & 0.177 & 0.233 \\
\hline 2 & 1 & $3.0-8.5$ & 0.139 & 0.165 \\
\hline 2 & 0.5 & $5.0-10.0$ & 0.113 & 0.161 \\
\hline 0.5 & 8 & $1.6-4.3$ & 0.240 & 0.341 \\
\hline 0.5 & 4 & $1.9-4.3$ & 0.275 & 0.395 \\
\hline 0.5 & 2 & 1.94 .6 & 0.232 & 0.329 \\
\hline 0.5 & 1 & 2.44 .8 & 0.069 & 0.093 \\
\hline 0.5 & 0.5 & $3.0-7.0$ & 0.028 & 0.047 \\
\hline
\end{tabular}

${ }^{a}$ Blood concentrations during this time interval were used to estimate $V_{m}$ and $K_{m}$ using a linear transformation of the Michaelis-Menten equation (see Appendix).

compartment open model are fairly close to the value of $\beta,{ }^{3}$ the apparent elimination rate constant of the two-compartment open model. (c) The value of $V_{m}$ estimated by one-compartment analysis is always less than the $V_{m}$ of the two-compartment open model. (d) The value of $K_{m}$ may be appreciably overestimated or underestimated when two-compartment data are analyzed by the use of a one-compartment open model.

Nonlinear in vivo data have in the past almost exclusively been analyzed using one-compartment analysis. The above simulations illustrate that one-compartment analysis of real data, which "actually" obey the twocompartment model, would yield numbers having very little relationship to those which would be obtained by two-compartment analysis. In vitro enzyme constants are often determined by incubating purified enzyme with substrate in a well-stirred system. Such an in vitro system would be expected to obey the one-compartment open model. Thus enzyme parameters determined from in vitro experiments, by utilizing one-compartment analysis (e.g., Lineweaver-Burk plots), should accurately reflect the in vitro enzyme constants under the particular experimental conditions. Moreover, if

${ }^{3} \beta=1 / 2\left\{\left(k_{12}+k_{21}+{ }^{v} m / K_{m}\right)-\left[\left(k_{12}+k_{21}+{ }^{V} m / K_{m}\right)^{2}-4 \cdot k_{21} \cdot{ }^{v} m / K_{m}\right]^{1 / 2}\right\}$. For the examples in the text, $\beta=0.700$. 


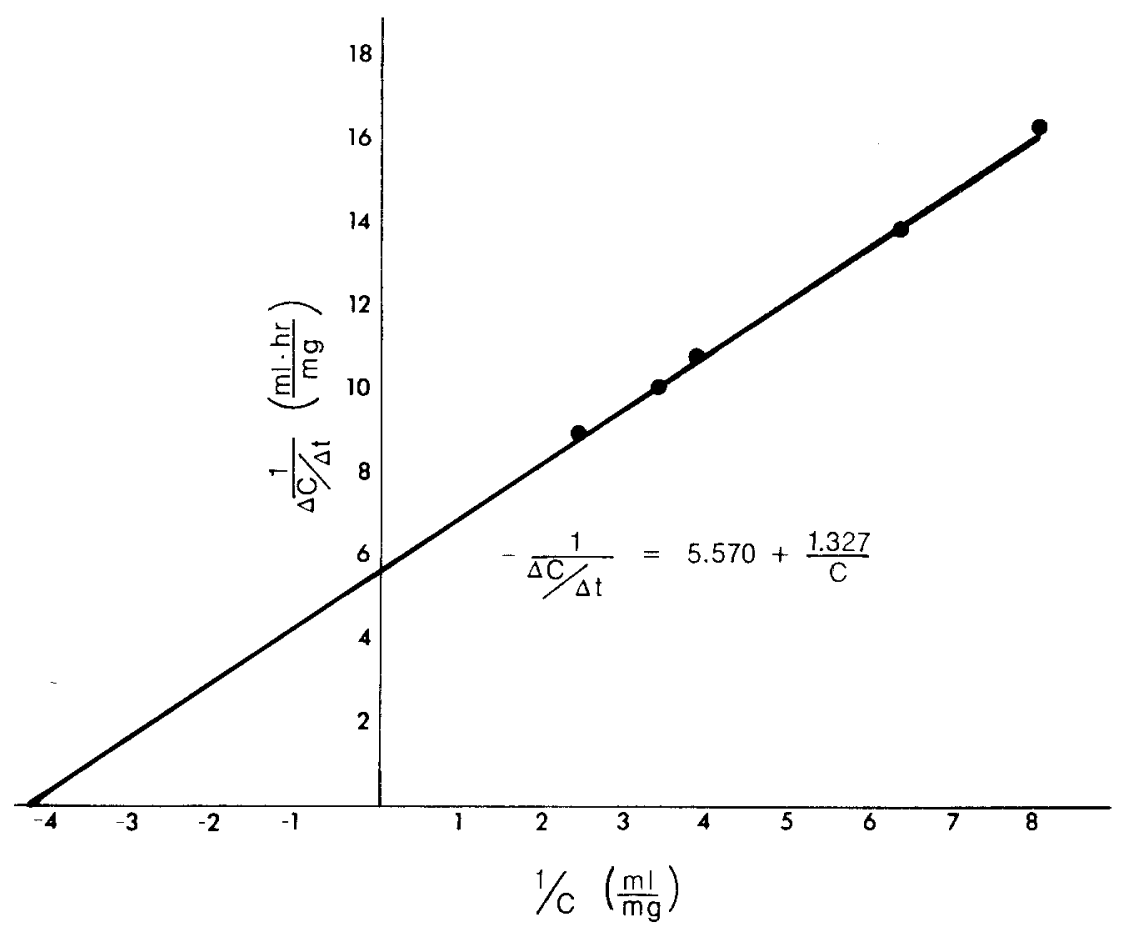

Fig. 6. Lineweaver-Burk plot of simulated blood concentration data which were generated from the two-compartment open model (scheme 1) with $k_{12}=3, k_{21}=2, k_{a}=8, C_{0}=2$, $V_{m}=0.418$, and $\dot{K}_{m}=0.182$.

in vitro experimental conditions accurately simulated in vivo enzyme environment, the values of these in vitro constants would be the same as the in vivo values. For drugs described by the two-compartment open model, in vivo enzyme constants obtained by one-compartment analysis do not agree well with the "true" values used in the simulations. Therefore, for these drugs, in vivo enzyme constants obtained by one-compartment analysis would not be expected to agree with values obtained in vitro. If there is agreement between in vivo and in vitro enzyme constants (both based on the one-compartment open model), it is feasible that this may be explained by the fact that either the one-compartment open model does adequately explain the in vivo data or the in vivo data are describable by a multicompartment model and the in vitro experimental conditions were fortuitously chosen in such a manner that agreement was obtained.

Decreases in relative bioavailability (based on area under blood $C, t$ curves) are often observed when drugs are administered at different doses and/or concomitantly with food. Classical pharmacokinetic models cannot 
easily explain these decreases for drugs which are fully absorbed. The oneand two-compartment open models with Michaelis-Menten elimination kinetics predict decreases in relative area under blood $C, t$ curves when smaller doses of drugs are administered or if the rate of drug absorption is slowed. The latter model may prove to be of value for describing the effect of food, etc., on bioavailability for drugs which distribute into a second compartment.

\section{CONCLUSION}

Values of apparent enzyme constants, $V_{m}$ and $K_{m}$, estimated by utilizing one-compartment analysis for drugs which are actually described by the two-compartment open model involving Michaelis-Menten elimination kinetics bear little relationship to the values of these constants applicable to the multicompartmental system. To distinguish a one-compartment from a multicompartmental system, the simulations discussed have indicated that blood levels need be measured after several different doses of a drug. At low doses there may be no "nose" on the blood concentration profile, whereas at higher doses the "nose" becomes prominent (see Figs. 1 and 2). Once such a "nose" is seen, the investigator should become aware that the appropriate model most probably is a multicompartmental one. It is this "nose," evident in the alcohol data reported by Wagner and Patel (3), which leads the authors to believe that pharmacokinetics of ethanol in man involves a multicompartmental rather than a one-compartment model. This is being pursued with intravenous studies in man and will be reported at a later date.

\section{APPENDIX}

Calculation of the Values of $V_{m}$ and $K_{m}$ Utilizing the One-Compartment Open Model and Terminal Data Obtained from Computer Simulations of the Two-Compartment Model with Michaelis-Menten Elimination Kinetics

The one-compartment model (scheme 2) is described mathematically by

$$
-\frac{d C}{d t}=\frac{V_{m} C}{K_{m}+C}
$$

If one assumes $\Delta C_{l}^{\prime} \Delta t \simeq d C / d t$, this equation can be rearranged to give

$$
\frac{C}{-\Delta C / \Delta t}=\frac{K_{m}}{V_{m}}+\frac{1}{V_{m}} \cdot C
$$


A Woolf plot (8) is a plot of $C /-(\Delta C / \Delta t)$ vs. $C$, where $C$ is the average concentration of drug during the time interval $(\Delta t)$. This gives a straight line having a slope of $1 / V_{m}$ and an intercept of $K_{m} / V_{m}$.

The following leads to a modification of the Woolf plot:

$$
-\frac{1}{C} \frac{d C}{d t}=-\frac{d \ln C}{d t}
$$

which rearranges to

$$
\frac{C}{d C / d t}=-\frac{d t}{d \ln C}
$$

Thus for two points, $n$ and $n+1$,

$$
\frac{C}{-\Delta C / \Delta t}=\frac{\Delta t}{-\Delta \ln C}=\frac{t_{n+1}-t_{n}}{\ln C_{n}-\ln C_{n+1}}
$$

Instead of using the arithmetic midpoint of $C_{n}$ and $C_{n+1}$ as the corresponding value of $C$, it is probably better to use the geometric mean of $C_{n}$ and $C_{n+1}$.

Hence for points $n$ and $n+1$,

$$
C=\left(C_{n} \cdot C_{n+1}\right)^{1 / 2}=e^{\left(\ln C_{n}+\ln C_{n+1}\right) / 2}
$$

Substituting equations 5 and 6 into equation 2 gives

$$
\frac{t_{n+1}-t_{n}}{\ln C_{n}-\ln C_{n+1}}=\frac{K_{m}}{V_{m}}+\frac{1}{V_{m}} \cdot\left(C_{n} \cdot C_{n+1}\right)^{1 / 2}
$$

Values of $y$ and $x$ were obtained by using simulated $C, t$ data. Linear regression of $y$ on $x$ gave an apparent straight line whose slope was $1 / V_{m}$ and intercept was $K_{m} / V_{m}$. These were solved to obtain estimates of $V_{m}$ and $K_{m}$.

\section{REFERENCES}

1. K. B. Bischoff and R. L. Dedrick. Thiopental pharmacokinetics. J. Pharm. Sci. 57: 13461351 (1968).

2. N. Gerber and J. G. Wagner. Explanation of dose-dependent decline of diphenylhydantoin plasma levels by fitting to the integrated form of the Michaelis-Menten equation. Res. Commun. Chem. Pathol. Pharmacol. 3: 455-466 (1968).

3. J. G. Wagner and J. A. Patel. Variations in absorption and elimination rate of ethyl alcohol in a single subject. Res. Commun. Chem. Pathol. Pharmacol. 4: 61-76 (1972).

4. G. Levy, T. Tsuchiya, and L. P. Amsel. Salicylate kinetics in man. Clin. Pharmacol. Therap. 13: $258-268(1972)$. 
5. J. P. Von Wartburg, J. L. Bethune, and B. L. Vallee. Liver alcohol dehydrogenase: Kinetic and physicochemical properties. Biochemistry 3: 1775-1782 (1964).

6. A. H. Blair and B. L. Vallee. Some catalytic properties of human liver alcohol dehydrogenase. Biochemistry 5: 2026-2034 (1966).

7. R. L. Dedrick and D. D. Forrester. Blood flow limitations in interpreting Michaelis constants for ethanol oxidation in vivo. Biochem. Pharmacol. 22: 1133-1140 (1973).

8. H. N. Christensen and G. A. Palmer. Enzyme Kinetics, Saunders, Philadelphia, 1967, pp. 101-103. 